\title{
Multidisciplinary Research Projects for Engineering Students
}

\author{
Ranjith A. Munasinghe \\ Department of Mathematics \\ West Virginia University Institute of Technology \\ Montgomery, WV 25136 \\ rmunasinghe@wvutech.edu
}

\section{Introduction}

Undergraduate engineering students learn calculus during the first three semesters in college. Most of the calculus topics are taught as abstract concepts without any science or engineering applications. It is not any better in other classes such as differential equations and linear algebra. Students are asked to solve algebraic and differential equations, integrate and differentiate functions, invert matrices, and sketch graphs of several types of functions.

Occasionally, we make them solve textbook application problems: They find volumes of solids, lengths of arcs, areas of surfaces, centroids of regions, and shortest distance from a point to a plane, etc. These so-called application problems are highly geometric in nature and designed for helping students to understand the concepts. They serve their purpose for the already motivated students. Many students raise the question in classrooms: What are the real applications? We do not discuss enough problems related to real engineering applications to show the students the connection between subject matter and the real world. Without making this connection it is difficult to emphasize the importance of learning fundamental principles of Mathematics. It is not possible to include many real applications in mathematics syllabi for a simple reason. The syllabi are too long and the students are not exposed to enough science and engineering concepts at the time they are taking lower level classes. In order to discuss real applications the instructor needs extra time to teach the engineering concept that is related to the problem. Perhaps, a complete reform of the way we teach mathematics to engineering students is necessary. We do not wish to discuss better ways to teach mathematics at this time. The goal of this paper is to provide resources and some ideas for undergraduate research projects and class projects suitable for mathematics and engineering students. Many teachers of mathematics, science, and engineering have identified research projects and class projects as important tools for motivating students to learn basic concepts. Research projects and class projects, if they are properly designed, can be the application problems we do not have time for in classrooms.

\section{What are the Properties of a Good Undergraduate Research Project?}

In order to identify properties of research projects suitable for undergraduates, we must 
first understand what we can and should expect from involving students in research projects. In addition to our primary goal, motivating students to learn basic mathematical and scientific principles, what else can we expect from research projects?

1. Research projects encourage independent thinking and learning. There are many forces associated with college education driving the teachers to encourage just a successful transfer of conventional knowledge and if not suppress, at the least neglect the importance of independent thinking.

2. How many times have we told our students that a certain topic is not in the syllabus and if we do not follow the syllabus, they would not be able to understand the material in the sequel to the class? Undergraduate projects are for the capable students interested in penetrating this unfortunate boundary.

3. Many upper classmen, specially the students we would select for research projects, entertain the idea of going to graduate school. Introduction to research by an undergraduate project can help them make this important career decision.

4. Obviously, research projects promote team work and writing project reports and presentations improve communication skills.

5. We must not forget the most fundamental goal of any research project. We conduct research to find new ideas and invent new things. In fact, undergraduate researchers have a unique advantage that sometimes could help them invent revolutionary solutions to complex problems. They have a fresh point of view. Their approach to problem solving is not completely guided by conventional education.

In order to achieve these goals an undergraduate research project must have the following properties.

1. A good undergraduate project is simple in nature. Participating students should be able to understand the research problem and the basic methods involved and start working on the project or at the least some aspects of the project without reading several research papers on the subject. After the students develop a deeper interest and meet with challenges they can be motivated to learn advanced mathematical tools related to the project and read research papers on the subject. 2. Real engineering problems are more desirable. The way the research problem is presented to the students could make a difference. For an example, consider a research project that involves stereo vision. One could make students take this project as a laboratory experiment; study the methods of constructing three-dimensional data from two images of an object from two different points of views. The same project would be more attractive to the students as a reverse engineering problem; construction of three-dimensional images of existing engineering structures for detecting deformations. We do not wish to undermine the importance of basic scientific and mathematical research. For the future mathematicians and the scientists we should provide necessary nourishment. We will discuss a project in mathematics that is suitable for mathematics majors and engineering students interested in learning more mathematics.

3. In addition to research work, the project should require students to publish papers, write project reports, and make presentations to the peers and teachers from their work.

4. Students will benefit more from a project that has the potential to grow to be his or her research work in graduate school. Such a project can change the career goals of a student and perhaps, seed a future scientist.

"Proceedings of the 2005 American Society for Engineering Education Annual Conference \& Exposition Copyright (C) 2005, American Society for Engineering Education" 


\section{Research Projects for Undergraduate Students}

\section{Image compression}

Two undergraduate students, Lesley and Brandy, worked on this project under the guidance of the author in the summer of 2004. The students found a method to compress bitmap images of people (identity photographs) by saving only the picture of the person without the background.

\section{Project Description}

Photographs used for identification such as photographs of suspicious individuals in police department files are often stored and transmitted electronically. Since a large number of such photographs are used by law enforcement agencies a slight compression of data will save a considerable amount of resources. In many cases, the backgrounds of these images do not contain any useful information. Students were directed to use this redundancy of data to find a method to compress the images. They were also directed to learn singular value decomposition (SVD) of matrices and applications of SVD to data compression.

\section{Project Outcomes}

Students learned a great deal on singular value decomposition of a matrix. They proved several theorems and learned how the best reduced rank approximation to an $m \times n$ matrix can be obtained using the SVD. (SVD is an example for an important tool used in advanced research that is an extension of a topic, diagonalization of symmetric matrices, in undergraduate curriculum. We refer the reader to an article by Kalman ${ }^{3}$ for details on theory and applications to data compression of SVD of matrices.) However the students were more interested in the application part of the project and we will describe their work on this problem in more details.

The solution was based on a simple idea: save a file that contains pixel information of the picture of the person without the background and upon retrieval of the file reproduce the image with a new generic background. Clearly, in addition to the pixel data of the picture of the person, it is necessary to save sufficient information regarding the locations of saved pixels. The very first difficulty the students faced was detecting the edge between the background and the person in the picture. They studied conventional edge detection and segmentation methods, tested them using Mathcad, and found that they do not produce satisfactory results. Mathcad can retrieve the pixel information of a bitmap image and provide tools for basic programming. Since these programs are easier to write and debug than regular computer programs, we believe that software packages such as Mathcad are more appropriate for the preliminary stages of a research project. After testing many ideas, they found a novel method to separate the portrait of a person from the background. The method produces excellent results for the images with fairly uniform backgrounds and no edges that separate the image into two regions in the background.

"Proceedings of the 2005 American Society for Engineering Education Annual Conference \& Exposition Copyright (C) 2005, American Society for Engineering Education" 
They called this method "Painting Technique" and it exploits the fact that the edge between the background and the person extends between two of the borders of the image and that it separates the image in to two regions. We show the results using Marvan's picture in Figure 1(a). Figure 1(b) shows the edge, detected by the program, between Marvan and the background. At this stage pixels belonging to Marvan's portrait are identified and in Figure 1(b) they are marked in gray.

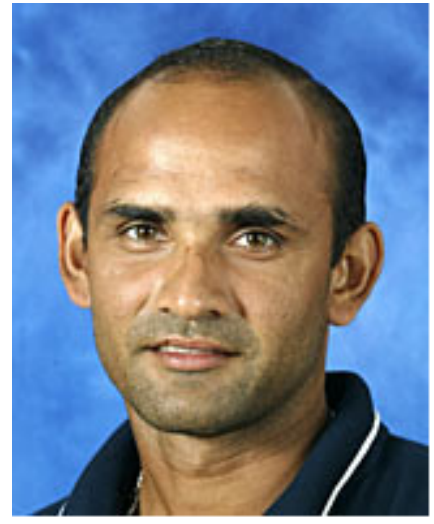

(a)

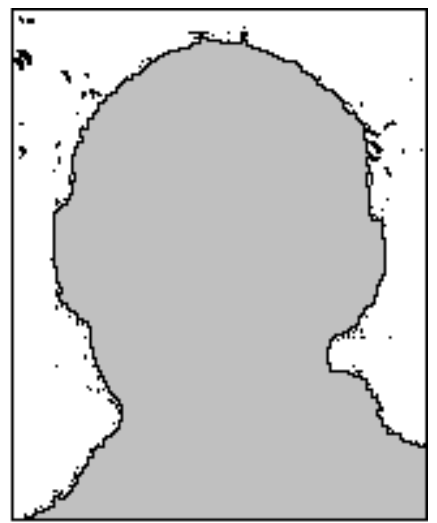

(b)

Figure 1

When the picture was stamped on a new background it looked like the picture was cutout and pasted. In order to solve this problem, in addition to gray pixels, a few pixels from the background along the edge were saved. Upon retrieval of the image, those background pixels were used to create a more natural and matching background for the image.

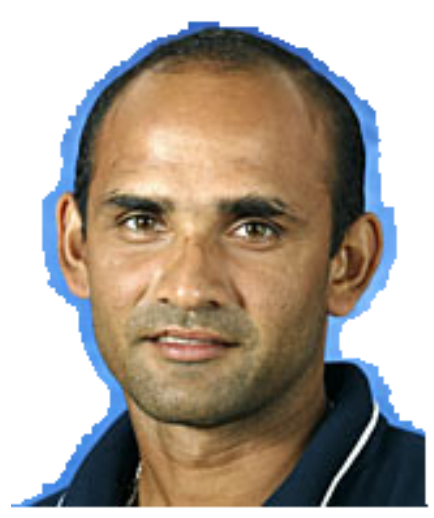

(a)

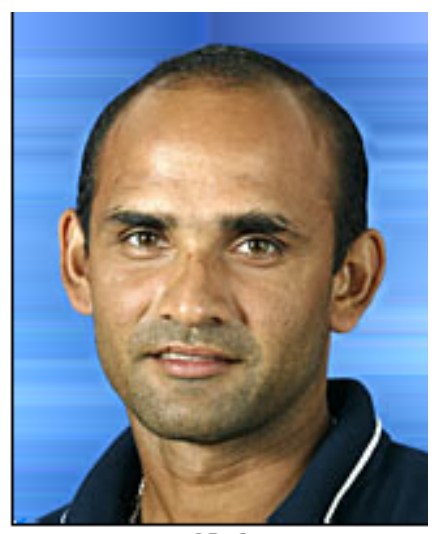

(b)

Figure 2

Figure 2(a) shows Marvan with the additional background pixels saved in the compressed

"Proceedings of the 2005 American Society for Engineering Education Annual Conference \& Exposition Copyright (C) 2005, American Society for Engineering Education" 
version of the image. Figure 2(b) shows Marvan with the background created by the program using few background pixels shown in Figure 2(a).

Students created a new file format to save the compressed version of the image. With the pixel information this version of the file contains a roadmap, an array in size equal to two thirds of the pixels in one column of the image, that is used to find the location of each pixel upon the retrieval of the file. The method was tested using several images and the results indicated that it yields a compression ratio of $30-40 \%$. In their project report ${ }^{1}$ and presentations students analyzed the results of the project and identified the limitations of their methods and future directions for the project. Their work is selected for presentation on the "Undergraduate Research Day at the Capital".

The students learn the following form the project: (a) Conventional edge detection and segmentation methods, (b) How to use Mathcad, (c) Bitmap file format and how to create a new file format, and (d) Singular value decomposition of matrices and their applications.

\section{Second Order Linear Differential equations with Variable Coefficients}

In elementary differential equations class we spend several days on theory of linear differential equations. But Euler's equation is the only linear differential equation with variable coefficients we solve at undergraduate level without using power series method. (Power series method can be used to find exact solutions for some simple equations. This method involves long calculations and the solutions are found in power series representation.) In this project we guide students to find other classes of linear equations with variable coefficients that can be solved without using infinite series method. This project is based on the author's published work ${ }^{4}$ and is suitable for a class project for a group of more advanced students. We present the project as a series of exercises. With very little guidance from an instructor, a student who has completed a first course in differential equations should be able to complete the exercises and discover solution methods for two classes of differential equations with variable coefficients.

\section{Project Problems}

First five exercises are fairly straightforward. The purpose of exercise 1 will be apparent after the exercise 5.

Exercise 1. Consider the linear differential equation $y^{\prime \prime}+p y^{\prime}+q y=0$ with constant coefficients $p$ and $q$. Let $c_{1}, c_{2}, c_{3}$ and $c_{4}$ be arbitrary real constants with $c_{1} c_{4}-c_{2} c_{3} \neq 0$. Show that $u(x)$, the ratio of independent solutions of the equation, takes one of the following three forms.

Case I: If $p^{2}-4 q<0$, then $u(x)=\frac{c_{1}+c_{2} \tan (a x)}{c_{3}+c_{4} \tan (a x)}$ where $a$ is a constant.

Case II: If $p^{2}-4 q>0$, then $u(x)=\frac{c_{1}+c_{2} e^{b x}}{c_{3}+c_{4} e^{b x}}$ where $b$ is a constant. 
Case III: If $p^{2}-4 q=0$, then $u(x)=\frac{c_{1}+c_{2} x}{c_{3}+c_{4} x}$.

Suppose that $y=y_{1}(x)$ and $y=y_{2}(x)$ are independent solutions of the differential equation

$$
y^{\prime \prime}+P(x) y^{\prime}+Q(x) y=0
$$

and let $u(x)=\frac{y_{2}(x)}{y_{1}(x)}$.

Exercise 2. Show that $y_{1}=\exp \left(-\frac{1}{2} \int\left(P(\mathrm{x})+\frac{u^{\prime \prime}}{u^{\prime}}\right) d x\right)$ and $y_{2}=u \exp \left(-\frac{1}{2} \int\left(P(\mathrm{x})+\frac{u^{\prime \prime}}{u^{\prime}}\right) d x\right)$ express the independent solutions of (1) in terms of the derivatives of $u$.

Exercise 3. The equation $w=\frac{u^{\prime \prime}}{u^{\prime}}$ is a first order variable separable equation in $u^{\prime}$. Solve the equation and express $u$ in terms of $w$. (Find the solution in most general form.)

Exercise 4. Show that

$$
w^{2}-2 w^{\prime}=2 P^{\prime}+P^{2}-4 Q .
$$

In what follows the function $D(\mathrm{x})=2 P^{\prime}(\mathrm{x})+P(\mathrm{x})^{2}-4 Q(\mathrm{x})$ is called the discriminant of the equation (1). The nonlinear differential equation (2) is an example of a Riccati equation, the equation that has the general form $y^{\prime}+a(x) y+b(x) y^{2}=c(x)$. The relationship between the second order linear differential equation and the Riccati equation is well known ${ }^{2}$. This equation can be solved when the discriminant of (1) has special forms.

Exercise 5. Assume that $D(x)=4 k$, where $k$ is a constant. Then the equation (2) becomes $w^{2}-2 w^{\prime}=4 k$ and is variable separable. Solve the equation and show that

Case I: If $k<0, u(x)=\frac{c_{1}+c_{2} \tan (\sqrt{-k} x)}{c_{3}+c_{4} \tan (\sqrt{-k} x)}$,

Case II: When $k>0, u(x)=\frac{c_{1}+c_{2} e^{2 \sqrt{k} x}}{c_{3}+c_{4} e^{2 \sqrt{k} x}}$, and

Case III: When $k=0, u(x)=\frac{c_{1}+c_{2} x}{c_{3}+c_{4} x}$ where $c_{1}, c_{2}, c_{3}$ and $c_{4}$ are arbitrary real constants with $c_{1} c_{4}-c_{2} c_{3} \neq 0$.

The ratios of independent solutions for the linear differential equations with constant discriminant and the ratios in exercise 1 , the ratios of independent solutions for the equations with constant coefficients, have identical forms.

Exercise 6. Using above exercises, outline a solution method for linear differential equations with constant discriminant.

Exercise 7. Show that the following linear differential equations have constant discriminant and find general solutions for them.

"Proceedings of the 2005 American Society for Engineering Education Annual Conference \& Exposition Copyright (C) 2005, American Society for Engineering Education" 

(a) $y^{\prime \prime}-4 y^{\prime} \tan 2 x-5 y=0$
(b) $y^{\prime \prime}+2 e^{x} y^{\prime}+\left(e^{x}+e^{2 x}\right) y=0$
(c) $y^{\prime \prime}+\frac{2}{1+x} y^{\prime}+9 y=0$
(d) $y^{\prime \prime}+\frac{2}{x} y^{\prime}-2 y=0$

The research work begins with the next exercise.

Exercise 8 . The equations with constant discriminant form a parent class for the equations with constant coefficients.

(a) Find the form of the discriminant of equations that generalizes Euler's equation in the same sense.

(b) Outline a solution method for the class of equations you found in (a).

(c) Can you find other classes of linear differential equations with variable coefficients that can be solved using this technique?

\section{Computer animations}

This idea for an interesting class project is an extension of a workshop conducted by the author for high school students. Concepts in multi-variable calculus topics such as vector valued functions, quadric surfaces, parametric equations of surfaces, and spherical and cylindrical coordinates are highly geometric in nature. Multi-variable calculus students often show difficulty in visualizing curves, surfaces, and regions in three dimensional space. We can use their fascination with computer graphics to help them overcome this difficulty. Using the tools in the calculus topics mentioned above, it is possible to create complex three dimensional animations. We propose to use Mathcad or another comparable software package to create and display animations. Mathcad graphic tools have many limitations. But Mathcad is easier to learn and use and therefore the student can concentrate on the mathematics of the animation. Perhaps, the limitations of the software package will motivate them to learn more computer programming and graphics.

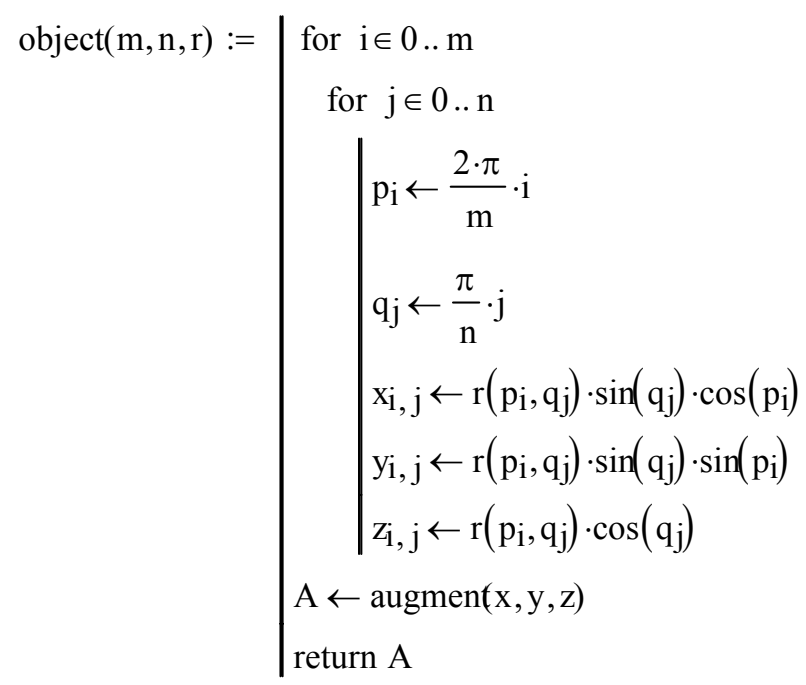

Figure 3

"Proceedings of the 2005 American Society for Engineering Education Annual Conference \& Exposition Copyright (C) 2005, American Society for Engineering Education" 
One way to create a surface plots in Mathcad is to create three arrays $x, y$, and $z$ such that for each $i$ and $j,\left(x_{i j}, y_{i, j}, z_{i j}\right)$ is a point on the surface. For an example, when $r(p, q)=1$ the routine in Figure 3 creates a matrix $A=[x|y| z]$ where the submatrices $x, y$, and $z$ contain $m n$ points on the unit sphere. (Unfortunately, Mathcad routines can return only one object and therefore it is necessary to augment $x, y$, and $z$ in to the matrix $A$ and after initializing $A$ recalculate $x, y$, and $z$ using Mathcad's submatrix function.) Notice that the routine uses spherical coordinates to form parametric equations for the surface given by $\rho=r(\theta, \phi)$. When $r(p, q)$ satisfies the condition that $r(p, q)=r(p+2 \pi, q+\pi)$ for all $p, q$ it creates a surface of a solid object similar to the asteroid in the Figure 5. The routine shown in Figure 4 combines rotations in three coordinate planes to rotate the object defined by the matrix $A=[x|y| z]$ about the x, $\mathrm{y}$, and $\mathrm{z}$ axes by angles all, al2, and al3, respectively.

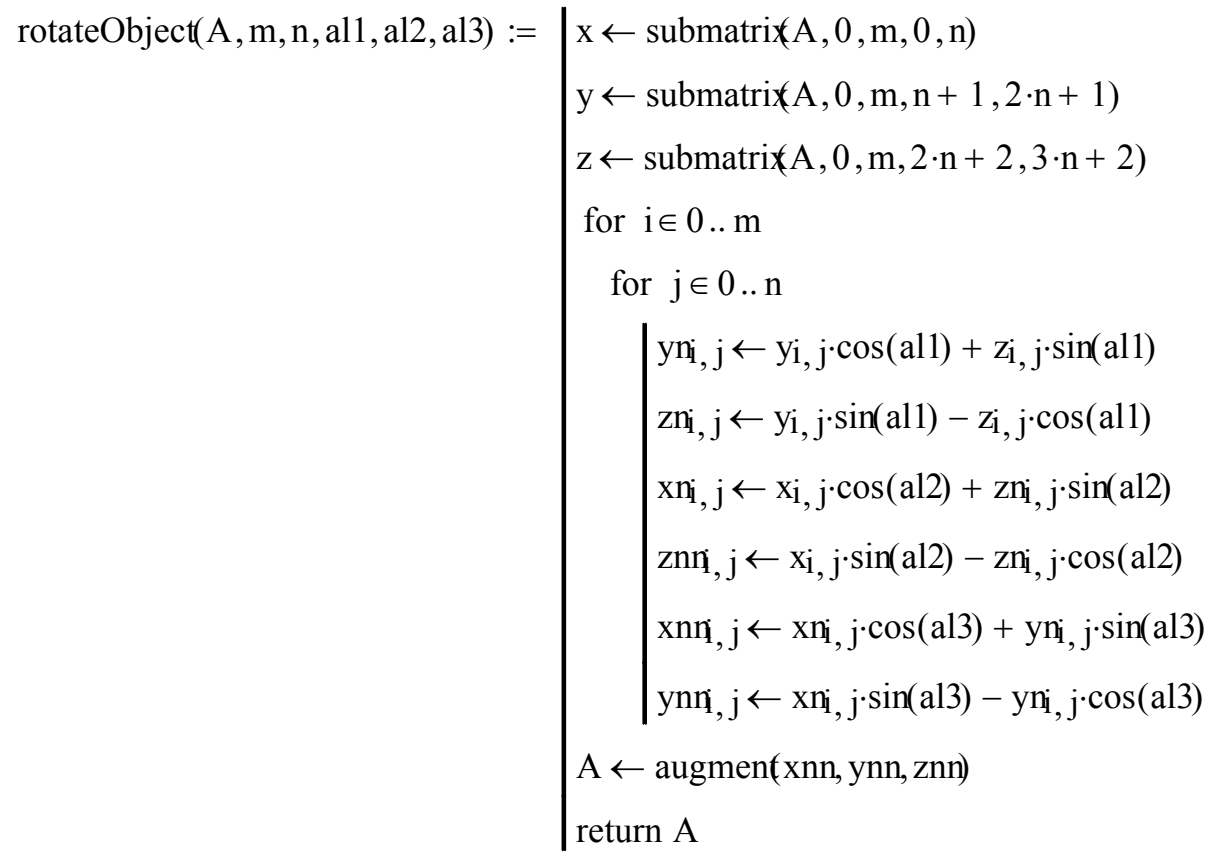

Figure 4

In Mathcad, animation is achieved by FRAME variable dependent objects. For example, after creating three arrays $x, y$, and $z$ for a sphere, adding $d$ times FRAME to each entry of the array $x$ creates a sphere that moves along the $x$ axis by $d$ units as the value of FRAME varies from one integer to the next. Figure 5 shows several frames from an animation, displaying the collision of an asteroid with a planet, created using these two routines. The next example uses a transparent surface to create the illusion of water. 

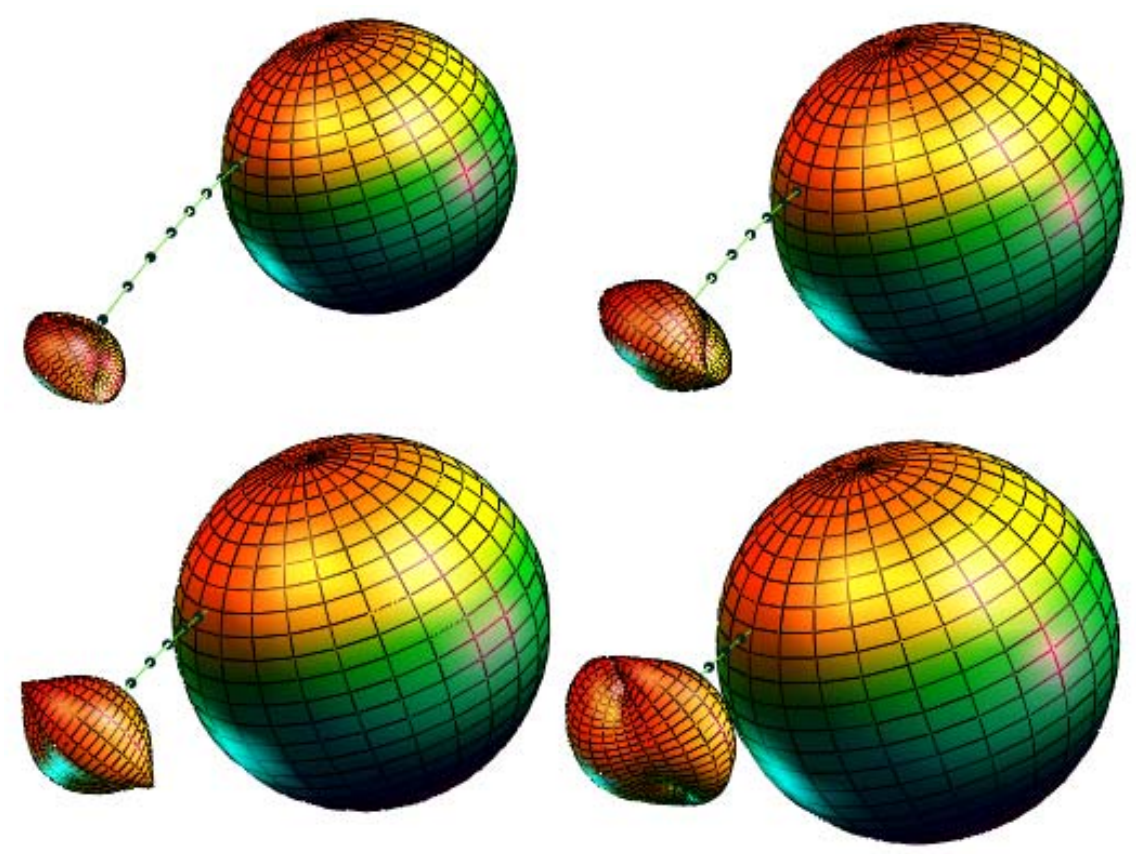

Figure 5
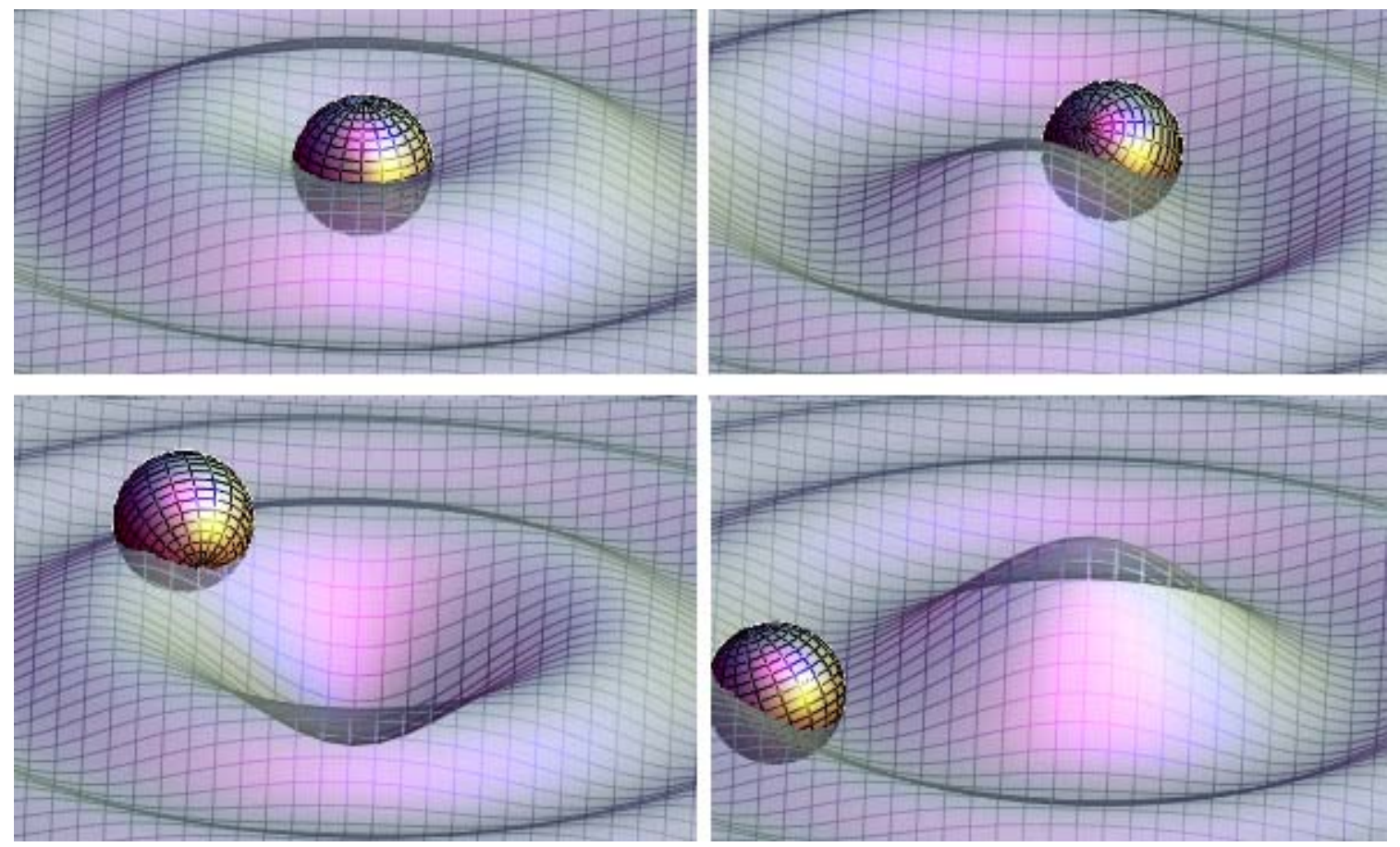

Figure 6

"Proceedings of the 2005 American Society for Engineering Education Annual Conference \& Exposition Copyright (C) 2005, American Society for Engineering Education" 
In the animation shown in Figure 6, the ripples are created by changing the phase angle of the function given by $f(x, y)=\sin \left(x^{2}+y^{2}\right)$. The ball moves out while rotating in a random manner along a spiral. Both animations are created with more than 50 frames and Mathcad allows animations with several hundred frames. Animations must be created so that they can be modified easily. For example, in both animations the paths of the moving objects can be changed just by editing the equations of the paths. Adding more like-objects is equally easy. We invite the reader to view more complex animations created using Mathcad at the author's website 5 . Complexity of the animations that can be created using multi-variable calculus is only limited by student's imagination. For this project there is no strict problem definition. The instructor could show the students a few simple animations similar to above examples and direct students to create animations of their choice.

Research projects and class projects can play an important role in undergraduate engineering education and we close with that brief conclusion.

\section{Bibliography}

1. Lesley Cavalier and Brandi Shafer, Image Compression by Deleting the Background, Project Report, College of Engineering, WVU Inst. of Tech., Montgomery, WV 25136, August 2004

2. Harold Davis, Introduction to Nonlinear Differential and Integral Equations, Dover, 1963

3. Dan Kalman, A Singularly Valuable Decomposition: The SVD of a Matrix, The College Mathematics Journal, Vol. 27, No. 1, January 1996.

4. Ranjith Munasinghe, Some Linear differential Equations Forget That They Have Variable Coefficients, The College Mathematics Journal, Vol 35, No. 1, January, 2004

5. Ranjith Munasinghe, Animations, www.wvutech.edu/faculty/rmunasinghe/animations.html, 2004

\section{RANJITH MUNASINGHE}

Ranjith Munasinghe is Professor of Mathematics at West Virginia University Institute of Technology. He received a $\mathrm{Ph} . \mathrm{D}$. degree in Mathematics from University of Wyoming in 1992. His research interests include topological dynamical systems, theory of continua, machine vision, and image processing.

"Proceedings of the 2005 American Society for Engineering Education Annual Conference \& Exposition Copyright (C) 2005, American Society for Engineering Education" 